\title{
Dimitri EL MURR (éd.), La Mesure du Savoir : études sur le Théétète de Platon
}

Jean-Luc Périllié

\section{(2) OpenEdition}

Journals

Édition électronique

URL : https://journals.openedition.org/philosant/845

DOI : 10.4000/philosant.845

ISSN : 2648-2789

Éditeur

Éditions Vrin

Édition imprimée

Date de publication : 1 novembre 2014

Pagination : $334-338$

ISBN : 978-2-7574-0855-

ISSN : $1634-4561$

Référence électronique

Jean-Luc Périllié, «Dimitri el murR (éd.), La Mesure du Savoir : études sur le Théétète de Platon»,

Philosophie antique [En ligne], 14 | 2014, mis en ligne le 01 novembre 2018, consulté le 02 décembre 2022. URL : http://journals.openedition.org/philosant/845; DOI : https://doi.org/10.4000/philosant. 845

\section{(c) $)(1) \Theta$}

Creative Commons - Attribution - Pas d'Utilisation Commerciale - Pas de Modification 4.0 International - CC BY-NC-ND 4.0

https://creativecommons.org/licenses/by-nc-nd/4.0/ 
celui-ci apparaissant tantôt comme un obstacle à la connaissance et tantôt comme un véhicule de l'âme nous permettant de nous élever vers le divin, M. A. Fierro (« Two conceptions of the body in Plato's Phaedrus », p. 27-50) propose une explication fondée sur la différence entre les projets et les objectifs dialectiques de chaque dialogue et souligne que ces différentes approches peuvent également être combinées au sein d'un dialogue unique, ce qui se produit dans le Phèdre. Quant à T. Johansen («Timaeus in the cave», p. 90-109), il met au jour les connexions profondes entre la République et le Timée, la première, selon la formule des anciens commentateurs, disant « de manière éthique » ce que le second dit « de manière physique », chacun des deux aspects étant néanmoins présent dans les deux dialogues, manifestant ainsi les connexions essentielles entre la sphère humaine et le reste du cosmos.

Ces quelques remarques ne visent aucunement à dresser le portrait complet d'une méthode unitaire qui serait partagée par l'ensemble des contributeurs de ce volume. Elles cherchent seulement à suggérer la fécondité du type d'approche dont Chr. Rowe est l'un des défenseurs les plus actifs et les plus éminents, et par conséquent la richesse de ce très beau volume qui lui est offert, dont les contributions de haut niveau ne manqueront pas de stimuler la réflexion de tous les amoureux de Platon.

Sylvain DELCOMMINETTE Université Libre de Bruxelles

Dimitri El MURR (éd.), La Mesure du Savoir: études sur le Théétète de Platon, Paris, Librairie philosophique J. Vrin, 2013 (Tradition de la pensée classique), 426 pages, ISBN 978-2-7116-2495-9.

La première étude de ce substantiel recueil de travaux savants sur le Théétète est signée de Marc-Antoine Gavray. L'objectif est de faire apparaître, à l'occasion de la citation du livre La Vérité de Protagoras, la leçon d'herméneutique qui se met en place, excédant le simple cadre de la réfutation. Lire en philosophe, ce n'est pas simplement expliquer, analyser, saisir les présupposés de la thèse, mais la comprendre: «L'herméneutique platonicienne se présente (...) comme la recherche d'une collaboration en vue d'un cheminement commun qui postule la diffusion de l'écrit, la notoriété de son auteur, l'intérêt de son contenu et la possibilité de l'accompagner dans le raisonnement (...) » (p. 28). La formule est intéressante mais peut porter à confusion, car M.-A. Gavray prend la diffusion du livre comme une donnée allant de soi, non problématique à la base. Dans le Théétète, la référence au livre La Vérité (162a) n'est effective que parce qu'elle est replacée dans le contexte de l'examen du «nouveau-né » de Théétète, précisément au cours de la procédure de l'amphidromie comme «promenade» du rejeton (160e-161a). En conséquence, ce qui est examiné c'est moins le livre de Protagoras que le fruit qui en résulte chez un lecteur particulier, en l'occurrence Théétète. Car, bien évidemment, le jeune homme a été ensemencé par sa lecture. Malheureusement, la caractéristique spermatique du «fragment» (p.28) n'a pas été relevée, alors que la courte citation de l'homme-mesure a pu produire tout ses effets... Il est pourtant question de sperma dans le discours maïeutique du Théétète (149e4). Il en résulte 
qu'il s'agit moins de s'ouvrir à l'herméneutique d'un livre (ce que pourrait faussement laisser supposer l'analyse de M.-A. Gavray) que de procéder à un examen maïeutique à caractère fondamentalement oral, examinant toutes les étapes d'une gestation. Vu sous cet angle, le Théétète doit être compris comme se situant dans le prolongement du passage du Phèdre concernant l'analogie des jardins d'Adonis structure valorisant la transmission orale et posant le réel problème de la valeur de l'ensemencement à partir de la lecture (276b-277a). Notons d'ailleurs que la question du sauvetage du discours (boethein to(i) logo(i)), autre procédure orale mise en évidence dans le Phèdre (275e, 276c), réapparaît explicitement dans le Théétète lorsque Socrate déplore que le mythos soit orphelin, que le père du discours ne soit pas là pour le défendre (164e, cité p. 31).

L'étude de S. Delcomminette aborde la partie finale de la réfutation de la première définition. En premier lieu l'auteur s'étonne de voir apparaître le thème de la paideia en 186b11-c5, quoique en réalité une telle émergence ne soit pas si surprenante dans le plus maïeutique des dialogues socratiques de Platon. Il convient alors de distinguer sensations et raisonnements qui confrontent les impressions dans leurs rapports à l'être et à l'utile. Si l'éducation platonicienne joue un rôle dans la perception, ce n'est pas en agissant directement sur la sensation, comme c'était le cas avec Protagoras (le sophiste modifiant l'insertion dans le monde de son élève en modifiant son système des croyances et des valeurs), mais en agissant sur la formation de la doxa dans sa différence avec la sensation. Il est question de l'éducation de la perception dans les livres VI et VII de la République, notamment dans le «mythe de la Caverne » selon la procédure de la periagoge. S. Delcomminette montre alors que le Théétète à son tour reprend le thème pédagogique dans la Digression, en établissant une distinction plus nette entre sensation et apparence. Si l'approche «génétique » République-Théétète est convaincante, nous ne pouvons en revanche qu'être réservé envers les appréciations finales, lorsque l'auteur affirme que, selon cette éducation-ci, «le bonheur ne se trouverait nulle part ailleurs que dans cette recherche qui n'a pas de fin ». Le philosophe trouverait-il alors son bonheur dans les perplexités de l'aporia? En bref, on pressent dans cette remarque la projection d'une conception positive de l'infini contraire aux textes et pour le moins anachronique au regard de la plus stricte pensée platonicienne.

Dans son étude « Pourquoi l'erreur ? » ouvrant sur la deuxième définition de Théétète, Michel Narcy aborde la question du sens même de la doxa et du verbe doxazein. Il faut bien traduire doxazein par «opiner » puisque Théétète n'a pas vraiment compris ce que Socrate maïeutiquement lui suggérait en employant les verbes dianoesthai, episkepsasthai, krinein. M. Narcy montre alors que la deuxième partie du dialogue est une succession de cinq hypothèses portant sur la question de l'erreur, en vue de vérifier si Théétète a bien à l'esprit ce que Socrate espère voir sortir de sa bouche (p. 102), à savoir que si la science n'est pas sensation, elle est dianoia, discursivité, jugement, impliquant la possibilité de l'erreur. Les hypothèses proposées alors d'une manière absurde contiennent des éléments de solution que le jeune homme doit actualiser par lui-même : introduire les intermédiaires entre le savoir et le non-savoir, comme dans le Ménon, poser une position intermédiaire entre l'être et le non-être, comme le fera l'Étranger du Sophiste. De cette analyse il 
découle que le Socrate du Théétète n'est pas un guide (p. 106-108) mais un testeur, conformément à la techne maieutike. Mais comme Théétète n'a pas su saisir au vol les perches qui lui étaient tendues, Socrate, le lendemain de l'entretien, n'aura d'autre solution que de « jouer les entremetteurs » en confiant le jeune homme à l'Étranger, activant à cet égard un nouvel ergon de la maieutike techne. Cette étude offre ainsi l'avantage de replacer l'œuvre entière et même le début de l'œuvre suivante (le Sophiste) dans le cadre global de la maïeutique. M. Narcy, dans l'introduction de sa traduction, voyait le Théétète comme le dialogue de clôture du cycle apologétique consacré à Socrate. Le Théétète serait donc bien un dialogue récapitulatif sur Socrate. Or Platon avait déjà substitué dans le Ménon (p. 108) et les dialogues de période médiane la nouvelle fonction, celle de dialecticien-guide, à la fonction originairement socratique de dialecticien-maïeuticien, impliquant l'inscience ou la rétention du savoir. Dommage que M. Narcy n'ait pas fait remarquer que le Socrate testeur (avec Hippocrate) et entremetteur - donc maïeuticien - était bien anciennement à l'œuvre dans le Protagoras, comme l'a montré Julius Tomin. Voir aussi le Lachès, 200c-d. De même Socrate, dans le Banquet de Xénophon, se définit comme entremetteur, allant même jusqu'à employer le terme de proxénète (mastropos).

L'étude de Monique Dixsaut établit que l'«opinion vraie »du Théétète ne peut jamais entrer pour partie dans la définition de la science. Non seulement la réfutation de la troisième définition révèle que l'addition du logos à l'opinion vraie aboutit toujours à un résultat nul (car ce que l'on ajoute à l'opinion vraie, elle l'avait déjà), mais en plus aucun logos du Théétète ne peut être identifié au « raisonnement sur la cause » du Ménon. À la fin de son étude, M. Dixsaut montre que la formule du Ménon vaut pour les sciences dianoétiques, car au moyen de la réminiscence la transformation des opinions vraies en connaissances (epistemai) est possible (p. 148). Mais elle ne peut valoir pour le logos dialectique dont le rapport à la science ne peut pas être de l'ordre de la possession mais seulement du désir amoureux (Rép. V, 485b). «Les modalités du jugement (...) qui immunisent contre l'inquiétude de la pensée [i.e. le jugement dianoétique] ne sont pas celles du discours dialectique », dit M. Dixsaut. Toujours est-il que l'approche spécialement « érotique » qui est celle des dialecticiens, si elle n'est pas de l'ordre de la possession, ne relève pas pour autant d'un amour infiniment lointain. Socrate (Rép. VI, 490b), à la manière de Diotime, parle explicitement de l'union du philosophe avec l'être véritable (migeis to(i) onti ontos), engendrant intellection et vérité. La philosophie platonicienne comme désir n'implique donc ni l'absence d'union ni par conséquent l'inquiétude de l'impuissance, d'autant plus qu'il y a bien « puissance $\gg . .$. Pourquoi donc ne pas reconnaître l'existence d'une dogmatique platonicienne, dès lors qu'il y a un réel engendrement de logoi issus de cette intime union avec l'être, ne pouvant évidemment être ramenés à la doxa, ni même à l'alethes doxa?

L'article suivant, de D. El Murr, reprend la même thématique en procédant à un examen détaillé de la réfutation dans le Théétète de la troisième définition de la science. Mais, avant cela, D. El Murr rapporte la vision du Commentateur anonyme médio-platonicien, qui voyait dans la maieutike techne la raison pour laquelle Socrate, dans le Théétète, laisse en suspens la formule du Ménon selon laquelle la 
connaissance diffère de l'opinion vraie par le fait qu'elle viendrait enchaîner les opinions vraies « par un raisonnement $(\log o s)$ sur la cause », formule qui aurait pu aboutir à la résolution de l'aporie finale. Malgré le caractère séduisant de la thèse de l'Anonyme, celle-ci ne sera pas retenue. La question est de savoir si le Théétète n'aurait pas soulevé une difficulté cruciale qui n’aurait pas été aperçue dans le Ménon. D. El Murr conclut par la négative car le Ménon fait apparaître un lien (desmos) qui est celui d'une âme qui désire connaître, et qui a saisi par la réminiscence les réalités intelligibles (p. 170). Il ne s'agit donc pas en parlant de «logos sur la cause » de rajouter une propriété objective à l'opinion droite.

L'étude de Chr. Rowe détonne en exposant une thèse totalement opposée à celle des contributeurs, contraire même à l'interprétation majoritaire du platonisme : l'aporie de la fin du Théétète serait seulement apparente et ne viserait qu'à corroborer l'insuffisance du savoir humain par opposition à la science divine : la définition finale serait en fait valable et vaudrait pour la science divine, si le logos ajouté à l'opinion vraie était pleinement assumé. L'aporie, par contre, correspondrait à la science imparfaite des hommes, ne disposant que de moyens limités pour produire le logos. La thèse est audacieuse en ce sens qu'elle fait fi - Chr. Rowe en est pleinement conscient - de la différence fondamentale entre le domaine de l'opinion (le sensible) et celui de la science (l'intelligible). Il est vrai que la dialektike ne peut pas ne pas passer par l'examen des doxai. De deux choses l'une : soit la science apparaît lors du surgissement d'un objet autre après une discussion préparatoire assez prolongée concernant les doxai, soit la science correspond à un cheminement graduel à partir des opinions vers la vérité. Le passage de Rép. VII, 534c correspondrait au second cas, alors que celui de la Lettre VII, 341c-d, correspondrait au premier. Mais comme, dans ce cas-ci, le passage d'une opération mentale à une autre reste obscur, on ne sait pas si on doit réellement distinguer les deux cas et si toutes les expressions relatives à un contact direct avec les Formes ne doivent pas rester seulement métaphoriques. Répondons que ce que décrivent au plus haut niveau à la fois la Caverne et la digression de la Lettre VII ne peut pas se ramener à la discursivité, ne peut pas relever seulement de la dianoia. Chr. Rowe voudrait ainsi réduire le système platonicien à une entreprise purement socratique, seulement discursive, selon les présupposés de son exégèse. Pourtant, il y a bien chez Platon une « puissance » qui dépasse les limitations de la discursivité, appelée noesis. La thèse de Chr. Rowe reste cependant intéressante en ce qu'elle met en évidence l'existence d'un système socratique perceptible au sein même de l'Apologie (20d23b) (cf. p. 175), opposant science humaine et science divine. Selon ce système, la science divine est inatteignable, seules les aletheis doxai peuvent s'en approcher, le philosophe pouvant dans une certaine mesure en rendre raison, produire dès lors un logos, celui-ci restant néanmoins scientifiquement insuffisant. Ce système réapparaît très clairement dans le Théétète comme dialogue récapitulatif, mais tout le monde voit bien, à l'exception de Chr. Rowe, qu'il ne résiste pas à l'aporie finale.

L'étude de F. Teisserenc montre, en effet, que l'aporie finale est non pas feinte mais bien réelle. Remarquons toutefois que cette étude, en dépit de son analyse détaillée des réfutations de la seconde et de la troisième définitions, place d'emblée ses analyses sur l'orbite des notions platoniciennes (Forme, participation) qui ne sont pas spécialement requises dans le Théétète. N'aurait-il pas été plus judicieux de 
laisser de côté ces notions, au moins dans un premier temps, pour mieux saisir en quelque sorte l'aporie à l'état brut, conformément à l'esprit de l'œuvre ? L'étude de F. Teisserenc, si elle tend à complexifier le problème, présente cependant un intérêt majeur : elle fait apparaître une faiblesse dans la thèse que l'on retient traditionnellement, thèse qui parvient à sa meilleure formulation avec Sedley, selon laquelle la notion de Forme en tant que non-dit du Théétète serait de nature à résoudre l'aporie finale. Or tel n'est pas le cas, selon F. Teisserenc. Ainsi le passage 146e qui pose le problème de savoir ce que l'episteme peut bien être en soi (gnonai epistemen auto hoti pot'estin) appellerait d'emblée une solution négative, car il n'y a pas d'en-soi de la science. F. Teisserenc montre ainsi que l'aporie finale ne résulte pas d'un simple changement de perspective (thèse de Rowe), qu'elle reste fondamentale et que sa solution réside moins dans la Forme que dans la notion de puissance (dynamis) (thèse de M. Dixsaut). Il faut dès lors se rapporter à République IV et V, notamment avec la dualité entre les pros alla et les auta kath'hauta - distinction qui débouche avec les pros alla sur la notion de dynamis. On sait d'ailleurs - F. Teisserenc aurait pu le rappeler - que cette dualité est très importante dans le système platonicien des agrapha dogmata. Force est donc de constater que cette solution dépasse la question originairement socratique du ti estin, ramenant naïvement la science à une essence. Teisserenc remarque alors d'une manière très subtile que le passage mathématique sur les puissances (dynameis) devait faire signe en direction de la solution, précisément la science comme puissance.

Il ne nous est pas possible, dans le cadre de ce compte rendu, de faire le point sur toutes les études. Aussi ne pouvons-nous qu'évoquer la seconde partie de l'ouvrage qui traite de l'importante postérité du Théétète. Sont examinés successivement les différentes versions du dialogue qui ont pu circuler dans l'antiquité (H. Tarrant), le rapport au stö̈cisme (A. Macé), la place du dialogue dans la classification de Thrasylle (D. Sedley), «l'unitarisme » de l'Anonyme médio-platonicien (M. Bonazzi), le rapport au plotinisme (S. Magrin) et à Montaigne (B. Sève). Ces études, toutes aussi passionnantes les unes que les autres, se prêtent cependant moins à la discussion. Ce qui a retenu spécialement notre attention dans cet ouvrage - qui fera certainement date dans les études sur le Théétète - réside dans son examen approfondi des questions relatives au caractère à la fois maïeutique et aporétique de ce grand dialogue.

Jean-Luc PÉRILLIÉ

Université Paul Valéry-Montpellier 3

Katja Maria Vogt, Belief and Truth. A Skeptic Reading of Plato, Oxford University Press, 2012, ix-209 pages, ISBN : 976-0-19-991681-8.

Cet ouvrage cherche à mettre en lumière et reconstituer une manière antique négligée d'analyser la connaissance que l'auteure désigne comme « socratique » (p. 3), comme «pyrrhonienne» (p. 184) ou comme une «lecture sceptique de Platon », et dont elle trouve et analyse les principaux éléments chez Platon, Sextus Empiricus et les stö̈ciens. Cette lignée est définie comme liant immédiatement les questions épistémologiques à des questions normatives à propos de notre incapacité d'examiner nos propres idées du fait de nos préférences (p. 3). L'une de ses thèses caractéristiques, selon Katja Vogt (désormais KV), est que la croyance (belief 